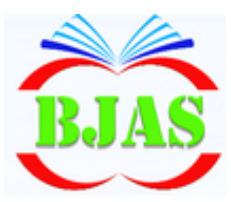

ISSN 1814 - 5868
Available online at http://.bajas.edu.iq http://dx.doi.org/10.37077/25200860.2019.194 College of Agriculture, University of Basrah

Basrah J. Agric. Sci., 32(2): 33-43, 2019

\section{Basrah Journal of Agricultural Sciences}

E-ISSN: 2520-0860

\title{
Prevalence and Antibiotic Resistance in Aeromonas species Isolated from Common Carp (Cyprinus carpio L.) Cultivated in Floating Cages at Al- Hilla River
}

\author{
Sadeq M. Al-Haider ${ }^{1}$, Khalidah S. Al-Niaeem ${ }^{2 *} \&$ Amjed K. Resen $^{2}$ \\ ${ }^{1}$ College of Veterinary Medicine, University of Al-Qasim Green, Iraq; \\ ${ }^{2}$ Department of Fisheries and Marine Resources, College of Agriculture, University of \\ Basrah, Basrah, Iraq
}

Received 15 March 2019; Accepted 24 May 2019; Available online 30 December 2019

*Corresponding author: kalidah_alsalim@yahoo.com

\begin{abstract}
The aim of this study is describe the case of ulcer and hemorrhaging (red-sore disease) in a common carp (C. carpio) that is cultivated in floating cages at Al-Hilla river and to finding efficient antibiotic treatments. A total of 144 specimens of the common carp were examined for ulcer with hemorrhaging during the period from December 2017 till the end of November 2018. In this study, red-sore disease was found in 45 specimens. This disease is caused by the infection of Motile Aeromonas Septicemia (MAS). Aeromonas hydrophila, A. sobria and $A$. veronii were isolated from the skin, gills and intestine of common carp. Aeromonas species were identified with a different percentage in common carp as the follows: A. hydrophila (58.76\%), A. sobria (31.83\%) and A. veronii (43.3\%). Antibiotic susceptibility test of 12 antibiotics (Piperacillin/ Tazobactan, Amikacin, Cefazolin, Gentamicin, Cefoxitin, Ciprofloxacin, Ceftazidime, Levofloxacin, Cefepime, Tigecycline, Imipenem and Trimethoprim/ Sulfamethoxazoleby) showed variable resistances for Aeromonas species. Aeromonas species were identified and examined for antibiotic susceptibility using the VITEK 2 system.
\end{abstract}

Keywords: Fish, Aeromonas spp., antibiotic susceptibility, Iraq.

\section{Introduction}

Aeromonas species cause a wide spectrum of disease syndromes among warm and cold blooded animals, including fish, reptiles, amphibians, mammals and humans (Janda \& Abbott, 2010). The members of Aeromonas spp. belong to the family Aeromonadaceae comprise Gram negative, nonspore forming and motile bacilli or coccobacilli rods with rounded ends (Skwor et al., 2014). It is cause most serious infectious disease known as Motile Aeromonas Septicemia (MAS), associated with high mortality rates without symptoms in per acute phase but in acute phase, skin and fin ulcers appear on the external surface of fish with ascites in the abdomen and exophthalmia (Bondad et al., 2005; Austin \& Austin, 2012; Skwor et al., 2014; Al-Niaeem et al., 2015). The disease 
can produce significant losses in aquaculture industry because of reduced growth in survived cases (Pachanawan et al., 2008; Buján et al., 2010). The Aeromonas spp. consist of 27 species, but some such as $A$. hydrophila, A. allosaccharophila, A. jandaei, A. piscicola, A. salmonicida, A. schubertii, A. bestiarum, A. sobria, A. veronii, A. dhakensis and $A$. caviae are considered pathogenic to animals and humans (Beazhidalgo \& Figueras, 2013; Aravenaromán et al., 2014; Li et al., 2019).

Outbreaks of the disease and its influence on the survival, persistence and ability of Aeromonas to cause infection are usually related with the change of environmental state, such as transfer the fish to poor water quality, stress, mishandling, changing of temperature, dioxide levels, overcrowding, high nitrite and carbon (Dixon, 1993; Aoki, 1999; Al-Taee et al., 2017).

Because, there is a little information pertains to Aeromonas in the fish farms particularly in Iraq. The aim of the present to describe a case of hemorrhaging and skin ulcer in common carp, C. carpio cultivated in floating cages in Al-Hilla river and the susceptibility pattern of bacteria to 12 antimicrobial drugs.

\section{Materials \& Methods}

A total of 144 fish of common carp were sampled from four farms of floating cages at Al-Hilla, the first and second stations, before the city center and the third and fourth stations, after the city center. During the period December 2017- November 2018. The length of fish ranging between $23.8-37.16 \mathrm{~cm}$ and the weight was 261.66- $693.3 \mathrm{gm}$. The live fishes were transported to oxygenated pond water. Before transferred to the laboratory in College of Veterinary Medicine, University of Al-Qasim Green.
The collected fishes were dissected and bacterial swabs were taken aseptically using a sterile loop from skin, gill and intestine. For isolation of bacteria, MacConkey Agar medium was used. The inoculated plate was incubated at $37{ }^{\circ} \mathrm{C}$ for $24 \mathrm{~h}$. Bacteria were identified and antibiotic susceptibility (Piperacillin/ Tazobactan, Amikacin, Cefazolin, Gentamicin, Cefoxitin, Ciprofloxacin, Ceftazidime, Levofloxacin, Cefepime, Tigecycline, Imipenem and Trimethoprim/ Sulfamethoxazoleby using the VITEK 2 system.

\section{Results \& Discussion}

The results of this study showed that $A$. hydrophila, A. sobria and A. veronii isolated and identified from the four floating cages in Al-Hilla river are a dangerous and may be unhealthy for public health when consumed. Aeromonas species were identified with different percentages for common carp, including A. hydrophila (58.76\%), A. sobria (31.83\%) and A. veronii (43.3\%) (Table 1). The Motile Aeromonas Septicemia is one that causes main disease problems in the carp farm (Cipriano, 2011). Outbreaks of the infectious disease are usually caused the changing of environmental state and stress. Fluctuation of temperature (sudden), crowding, poor water quality, low dissolved oxygen, high ammonia levels are the common factors related with MAS (Ko et al., 1998).

The study was conducted from December 2017 till the end of November 2018 in which there are fluctuations in water quality parameters in the aquaculture in the studied stations (Table 2). The mean of temperature fluctuated from $10.7{ }^{\circ} \mathrm{C}$ to $32.9^{\circ} \mathrm{C}$. the mean of salinity was recorded ranged from 460 to 580 PSU, ammonia was recorded ranged from 1.3 to $2.8 \mathrm{PSU}$. The $\mathrm{pH}$ value was relatively from 6.4 to 8.1 . In culture system of fish that 
always exposed an assortment of stresses become over sensitive to disease infection (Rijnsdorp et al., 2009; Albert \& Ransangan, 2013).

The growing of bacteria in water increased by rising levels of water temperature from 25 ${ }^{\circ} \mathrm{C}$ to $32^{\circ} \mathrm{C}$, organic matters, salinity and $\mathrm{pH}$
5-9 (Kiriratnikom et al., 2000). These levels of growing bacteria were observed in the current study.

Gram negative bacilli, A. hydrophila $A$. sobria and $A$. veronii were isolated from the skin, gills and intestine form common carp (Fig. $1 \&$ table 3).

Table (1): Percentage of isolated Aeromonas spp.

\begin{tabular}{|c|c|c|c|}
\hline Station & A. hydrophila (\%) & A. sobria $(\%)$ & A. veronii (\%) \\
\hline 1 & 11.76 & 5.88 & 9.80 \\
\hline 2 & 15.60 & 8.26 & 11.0 \\
\hline 3 & 14.40 & 7.69 & 11.5 \\
\hline 4 & 17.0 & 10.0 & 11.0 \\
\hline Total & 58.76 & 31.83 & 43.3 \\
\hline
\end{tabular}

Table (2): The water parameters of studied stations.

\begin{tabular}{ccccc}
\hline \multirow{2}{*}{ Station } & $\begin{array}{c}\text { Temp }\left({ }^{\circ} \mathrm{C}\right) \\
\text { Min-max (mean) }\end{array}$ & $\begin{array}{c}\text { Salinity (PSU) } \\
\text { Min-max (mean) }\end{array}$ & $\begin{array}{c}\left.\text { Ammonia (mg. }{ }^{-1}\right) \\
\text { Min-max (mean) }\end{array}$ & $\begin{array}{c}\text { pH } \\
\text { Min-max (mean) }\end{array}$ \\
\cline { 2 - 5 } & & \multicolumn{2}{c}{ Range (mean) } & \\
\hline 1 & $11.9-32.7(22.66)$ & $480-560(517.5)$ & $1.3-1.8(1.62)$ & $6.8-8.1(7.36)$ \\
\hline 2 & $10.7-32.9(22.9)$ & $460-570(516.66)$ & $1.4-1.9(1.69)$ & $6.5-8.1(7.33)$ \\
\hline 3 & $13-32.6(23.8)$ & $480-580(530.8)$ & $2.0-2.8(2.42)$ & $6.4-7.6(7.09)$ \\
\hline 4 & $12.5-31.2(22.86)$ & $460-560(513.33)$ & $0.7-1.4(1.21)$ & $7.1-7.9(7.35)$ \\
\hline
\end{tabular}

Biochemical characterization of Motile Aeromonas Septicemia using the VITEK 2 system was given in tables (4-6). It has been noticed by some researchers that Aeromonas spp. causes skin lesions with hemorrhagic in fresh water fish and cultured fish (Aguilera et al., 2005; Beazhidalgo \& Figueras, 2013; Skwor et al., 2014; Gao et al., 2016; Li et al., 2019).

The best antibiotics susceptibility were Tigecycline, Ceftazidime, Cefepime and Gentamicin, when tested in vitro on $A$. hydrophila while, it was resistant to Cefazolin and Cefriaxone (Table 7).

In vitro, the more antibiotic susceptibility to $A$. sobria were Ciprofloxacin, Levofloxacin, Tigecycline, Gentamicin, Cefazolin and Cefepime whereas $A$. sobria had intermediately resistant to Imipenem (Table 8). The antibiotic susceptibility in vitro were more effect on $A$. veronii Ciprofloxacin, Levofloxacin, Tigecycline, Gentamicin, Cefazolin and Cefepime (Table 9). 
Al-Haider et al./ Basrah J. Agric. Sci., 32 (2): 36-46, 2019
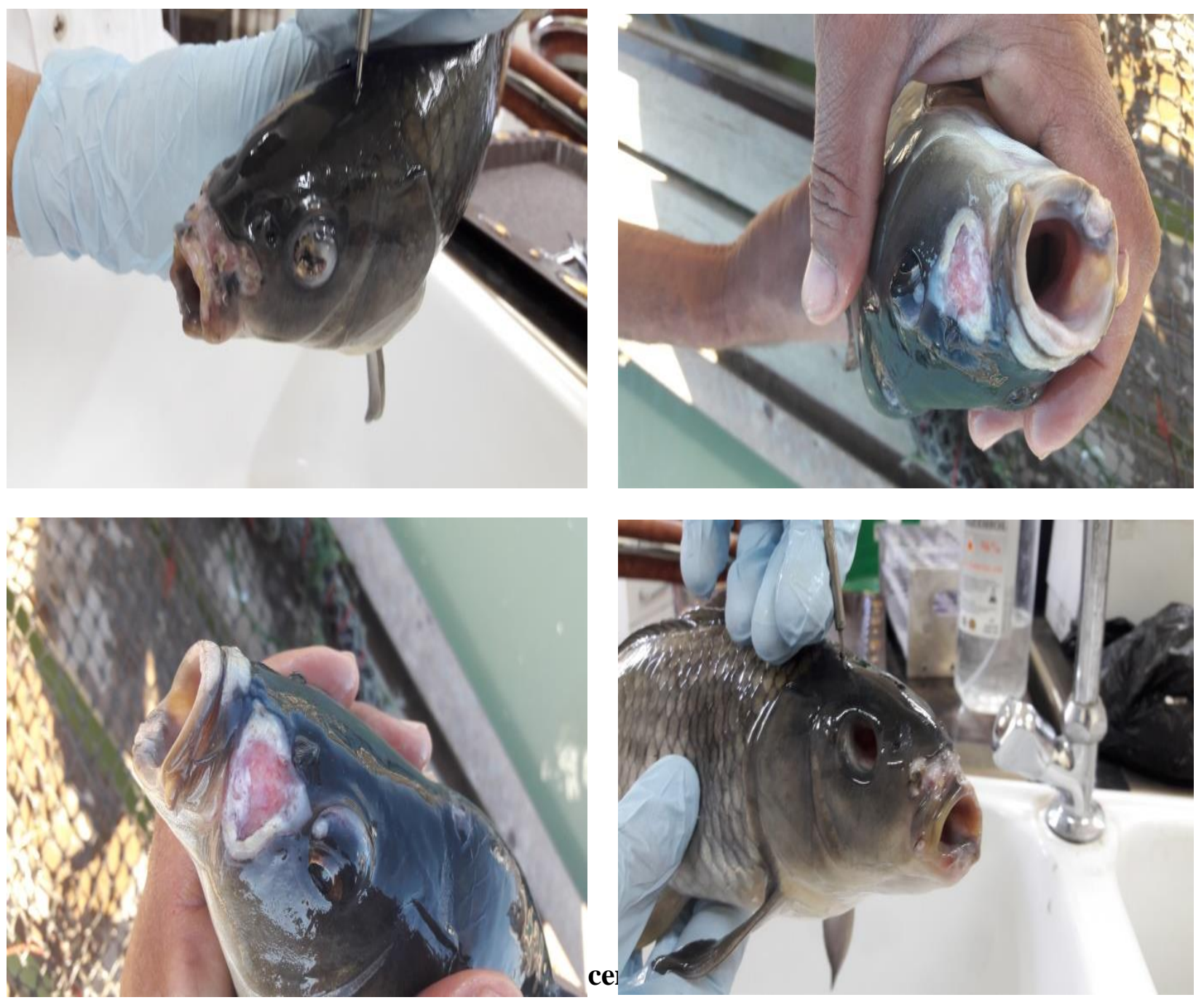
Al-Haider et al./ Basrah J. Agric. Sci., 32 (2): 36-46, 2019

Table (3): The infected organs of fish by Aeromonas spp.

\begin{tabular}{|c|c|c|c|c|c|c|c|c|c|}
\hline \multirow[t]{2}{*}{ Month } & \multicolumn{3}{|c|}{ Skin } & \multicolumn{3}{|c|}{ Gills } & \multicolumn{3}{|c|}{ Intestine } \\
\hline & A. hydrophila & A. sobria & A. veronii & A. hydrophila & A. sobria & A. veronii & A. hydrophila & A. sobria & A. veronii \\
\hline December 2017 & - & - & - & - & - & - & + & - & - \\
\hline January 2018 & + & + & - & - & + & - & - & + & - \\
\hline February & - & + & - & - & + & - & - & + & - \\
\hline March & + & + & - & + & - & - & + & + & + \\
\hline April & + & - & + & + & - & + & + & - & + \\
\hline May & + & - & + & + & - & + & + & - & + \\
\hline June & + & - & + & + & - & + & + & - & + \\
\hline July & + & + & + & - & + & + & - & + & - \\
\hline August & + & - & + & - & - & + & - & - & + \\
\hline September & + & - & + & + & - & + & + & - & + \\
\hline October & + & - & + & - & - & + & + & - & + \\
\hline November & - & - & + & - & + & + & + & - & + \\
\hline
\end{tabular}

+: Positive, -: Negative 
Al-Haider et al./ Basrah J. Agric. Sci., 32 (2): 36-46, 2019

Table (4): Biochemical features of isolated A. hydrophila.

Biochemical features Reactio Biochemical features Reactio

$\mathrm{n}$

$\mathrm{n}$

\begin{tabular}{|c|c|c|c|c|c|}
\hline 2 & APPA & - & 3 & $\mathrm{ADO}$ & - \\
\hline 4 & PyrA & - & 5 & IARL & - \\
\hline 7 & $\mathrm{dCEL}$ & + & 9 & BGAL & + \\
\hline 10 & $\mathrm{H} 2 \mathrm{~S}$ & - & 11 & BNAG & + \\
\hline 12 & AGLTp & - & 13 & dGLU & + \\
\hline 14 & GGT & - & 15 & OFF & + \\
\hline 17 & BGLU & - & 18 & dMAL & + \\
\hline 19 & dMAN & + & 02 & dMINE & + \\
\hline 01 & BXYL & - & 00 & BAlap & - \\
\hline 03 & ProA & - & 26 & LIP & + \\
\hline 07 & PLE & - & 09 & TyrA & + \\
\hline 31 & URE & - & 30 & dSOR & + \\
\hline 33 & SAC & + & 34 & dTAG & - \\
\hline 35 & dTRE & + & 36 & CIT & - \\
\hline 37 & MNT & - & 39 & $5 \mathrm{KG}$ & - \\
\hline 42 & ILATk & - & 41 & AGLU & - \\
\hline 40 & SUCT & + & 43 & NAGA & - \\
\hline 44 & AGAL & - & 45 & PHOS & + \\
\hline 46 & GIyA & - & 47 & ODC & - \\
\hline 48 & LDC & - & 53 & IHISa & - \\
\hline 56 & CMT & + & 57 & BGUR & - \\
\hline 58 & O129R & + & 59 & GGAA & + \\
\hline 61 & IMLTa & - & 60 & ELLM & + \\
\hline 64 & ILATa & & & - & \\
\hline
\end{tabular}

Table (5): Biochemical features of isolated A. sobria.

\begin{tabular}{llllll}
\hline \multicolumn{2}{l}{ Biochemical features } & Reaction & \multicolumn{2}{l}{ Biochemical features } & Reaction \\
\hline 2 & APPA & - & 3 & ADO & - \\
\hline 4 & PyrA & - & 5 & IARL & - \\
\hline 7 & dCEL & - & 9 & BGAL & + \\
\hline 10 & H2S & - & 11 & BNAG & + \\
\hline 12 & AGLTp & - & 13 & dGLU & + \\
\hline 14 & GGT & - & 15 & OFF & + \\
\hline 17 & BGLU & - & 18 & dMAL & + \\
\hline 19 & dMAN & + & 02 & dMINE & + \\
\hline 01 & BXYL & - & 00 & BAlap & - \\
\hline 03 & ProA & + & 26 & LIP & - \\
\hline 07 & PLE & - & 09 & TyrA & + \\
\hline 31 & URE & - & 30 & dSOR & - \\
\hline 33 & SAC & + & 34 & dTAG & - \\
\hline 35 & dTRE & + & 36 & CIT & + \\
\hline 37 & MNT & - & 39 & 5KG & - \\
\hline 42 & ILATk & - & 41 & AGLU & - \\
\hline
\end{tabular}


Al-Haider et al./ Basrah J. Agric. Sci., 32 (2): 36-46, 2019

\begin{tabular}{llllll}
\hline 40 & SUCT & + & 43 & NAGA & + \\
\hline 44 & AGAL & - & 45 & PHOS & - \\
\hline 46 & GIyA & - & 47 & ODC & - \\
\hline 48 & LDC & - & 53 & IHISa & - \\
\hline 56 & CMT & + & 57 & BGUR & - \\
\hline 58 & O129R & - & 59 & GGAA & + \\
\hline 61 & IMLTa & - & 60 & ELLM & + \\
\hline 64 & ILAT & - & & & \\
\hline
\end{tabular}

+: Positive, -: Negative

Table (6): Biochemical features of isolated $A$. veronii.

\begin{tabular}{|c|c|c|c|c|c|}
\hline \multicolumn{2}{|c|}{ Biochemical features } & \multirow{2}{*}{$\begin{array}{c}\text { Reaction } \\
-\end{array}$} & \multicolumn{2}{|c|}{ Biochemical features } & \multirow{2}{*}{$\begin{array}{c}\text { Reaction } \\
-\end{array}$} \\
\hline 2 & APPA & & 3 & $\mathrm{ADO}$ & \\
\hline 4 & PyrA & - & 5 & IARL & - \\
\hline 7 & dCEL & + & 9 & BGAL & + \\
\hline 10 & $\mathrm{H} 2 \mathrm{~S}$ & - & 11 & BNAG & + \\
\hline 12 & AGLTp & - & 13 & dGLU & + \\
\hline 14 & GGT & - & 15 & OFF & + \\
\hline 17 & BGLU & + & 18 & dMAL & + \\
\hline 19 & dMAN & + & 02 & dMINE & + \\
\hline 01 & BXYL & - & 00 & BAlap & - \\
\hline 03 & ProA & + & 26 & LIP & - \\
\hline 07 & PLE & - & 09 & TyrA & + \\
\hline 31 & URE & - & 30 & dSOR & - \\
\hline 33 & SAC & + & 34 & dTAG & - \\
\hline 35 & dTRE & + & 36 & CIT & + \\
\hline 37 & MNT & - & 39 & $5 \mathrm{KG}$ & - \\
\hline 42 & ILATk & - & 41 & AGLU & - \\
\hline 40 & SUCT & + & 43 & NAGA & - \\
\hline 44 & AGAL & + & 45 & PHOS & - \\
\hline 46 & GIyA & - & 47 & ODC & + \\
\hline 48 & LDC & - & 53 & IHISa & - \\
\hline 56 & CMT & + & 57 & BGUR & - \\
\hline 58 & O129R & - & 59 & GGAA & + \\
\hline 61 & IMLTa & - & 60 & ELLM & + \\
\hline 64 & ILATa & & & & \\
\hline
\end{tabular}

+: Positive, -: Negative

Table (7): Antibiotic susceptibility of $A$. hydrophila.

\begin{tabular}{lcclcc}
\hline \multicolumn{1}{c}{ Antimicrobial } & MIC & Interpretation & Antimicrobial & MIC & Interpretation \\
\hline $\begin{array}{l}\text { Piperacillin/ } \\
\text { Tazobactan }\end{array}$ & $<=4$ & $\mathrm{~S}$ & \multicolumn{1}{c}{ Amikacin } & $<=2$ & $\mathrm{~S}$ \\
\hline Cefazolin & $>=64$ & $\mathrm{R}$ & Gentamicin & $<=1$ & $\mathrm{~S}$ \\
\hline Cefoxitin & 32 & $\mathrm{R}$ & Ciprofloxacin & 1 & $\mathrm{~S}$ \\
\hline Ceftazidime & $<=1$ & $\mathrm{~S}$ & Levofloxacin & 1 & $\mathrm{~S}$ \\
\hline Cefepime & $<=1$ & $\mathrm{~S}$ & Tigecycline & $<=0.5$ & $\mathrm{~S}$ \\
\hline Imipenem & 4 & $\mathrm{~S}$ & $\begin{array}{l}\text { Trimethoprim/ } \\
\text { Sulfamethoxazole }\end{array}$ & $<=20$ & $\mathrm{~S}$ \\
\hline
\end{tabular}

MIC: Minimum Inhibitory Concentration ( $\mu \mathrm{g} \cdot \mathrm{ml}^{-1}$ ), S: Sensitive, R: Resistant. 
Table (8): Antibiotic susceptibility of $A$. sobria.

\begin{tabular}{lcclcc}
\hline \multicolumn{1}{c}{ Antimicrobial } & MIC & Interpretation & Antimicrobial & MIC & Interpretation \\
\hline $\begin{array}{l}\text { Piperacillin/ } \\
\text { Tazobactan }\end{array}$ & 16 & $\mathrm{~S}$ & Amikacin & $<=2$ & $\mathrm{~S}$ \\
\hline Cefazolin & $<=4$ & $\mathrm{~S}$ & Gentamicin & $<=1$ & $\mathrm{~S}$ \\
\hline Cefoxitin & $<=4$ & $\mathrm{~S}$ & Ciprofloxacin & $<=0.25$ & $\mathrm{~S}$ \\
\hline Ceftazidime & $<=1$ & $\mathrm{~S}$ & Levofloxacin & 0.5 & $\mathrm{~S}$ \\
\hline Cefepime & $<=1$ & $\mathrm{~S}$ & Tigecycline & $<=0.5$ & $\mathrm{~S}$ \\
\hline Imipenem & 8 & $\mathrm{I}$ & $\begin{array}{l}\text { Trimethoprim/ } \\
\text { Sulfamethoxazole }\end{array}$ & $<=20$ & $\mathrm{~S}$ \\
& & & & \\
\hline
\end{tabular}

MIC: Minimum Inhibitory Concentration ( $\left.\mu \mathrm{g} \cdot \mathrm{ml}^{-1}\right)$, S: Sensitive, R: Resistant, I: Intermediate.

Table (9): Antibiotic susceptibility of A. veronii.

\begin{tabular}{lcclcc}
\hline \multicolumn{1}{c}{ Antimicrobial } & MIC & Interpretation & Antimicrobial & MIC & Interpretation \\
\hline $\begin{array}{l}\text { Piperacillin/ } \\
\text { Tazobactan }\end{array}$ & 20 & $\mathrm{~S}$ & Amikacin & $<=2$ & $\mathrm{~S}$ \\
\hline Cefazolin & $<=4$ & $\mathrm{~S}$ & Gentamicin & $<=1$ & $\mathrm{~S}$ \\
\hline Cefoxitin & $<=4$ & $\mathrm{~S}$ & Ciprofloxacin & $<=0.5$ & $\mathrm{~S}$ \\
\hline Ceftazidime & $<=1$ & $\mathrm{~S}$ & Levofloxacin & 0.5 & $\mathrm{~S}$ \\
\hline Cefepime & $<=1$ & $\mathrm{~S}$ & Tigecycline & $<=0.5$ & $\mathrm{~S}$ \\
\hline Imipenem & 8 & $\mathrm{I}$ & $\begin{array}{l}\text { Trimethoprim/ } \\
\text { Sulfamethoxazole }\end{array}$ & $<=20$ & $\mathrm{~S}$ \\
\hline
\end{tabular}

MIC: Minimum Inhibitory Concentration ( $\left.\mu \mathrm{g} . \mathrm{ml}^{-1}\right)$, S: Sensitive, R: Resistant, I: Intermediate.

Recently, there are many processes for treating Aeromonas spp. (primarily $A$. hydrophila) that infected in cultivated fish, one of them is antimicrobial drugs, it is the common antibiotic for controlling the aquatic bacteria, so antimicrobial sensitivity experiments are crucial for an efficient therapy (Skwor et al., 2014).

High allergy of drug examination in various organisms is feasible technique that can clearly decrease antibiotic dosage (Skwor et al., 2014; Li et al., 2019). In the current study, the Motile Aeromonas Septicemia was intensely susceptible to Tigecycline, Ciprofloxacin and Levofloxacin. Therefore, it suggests may be that these drugs are convenient to treat Motile Aeromonas Septicemia. On the other hand, there are some risks for using antibiotics, firstly, the medicine can penetrate the biological membrane and tissues, for example, it can pass through blood vessels in the brain, secondly, using antibiotics for a long time causes the resistance of bacteria, drug accumulation in fish tissues and environmental problems (El-Bouhy et al., 2011; Li et al., 2019).

About $90 \%$ of A. hydrophila, were susceptible to Ceftazidine, Moxalactam, Aztreonam, Cefepime, Amikacin, Fluoroquinolones and Imipenem, but it was more resistant to TrimethoprimSulfameth- oxazole, Tetracycline, Aminoglycosides and Cephalosporins (Chopra, \& Roberts, 2001; Soltan et al., 2016). Guz \& Kozinska (2004) found that about 21 isolates of $A$. sobria and $A$. hydrophila which isolated from common carp were $100 \%$ were sensitive to Oxolinic acid, Trimethoprim- Sulphamides, 
Cloramphenicol, Flumequine, Norfloxacin, Perfloxacin, lincomycin and resistant to Penicillin.

Nawaz et al. (2006); Adanir \& Turutoglu (2007) and Hassan et al. (2017) confirmed that the resistance to Oxytetracycline, Penicillin, Amoxicillin and other antibiotics may be attributed to genes which recently discovered as responsible for this antibiotic resistance in genus Aeromonas.

\section{Conclusion}

Aeromonas spp. may cause hemorrhages with ulcers in common carp. Extensive and uncontrolled use of antimicrobial drugs may generate an multiple resistance for antimicrobial. So, the test of antimicrobials susceptibility in genus Aeromonas infection and other bacterial infections must be under intensive in cultivated fish. The water should be orderly changed and stress and overcrowding in the fish population should be avoided to prevent the infection.

\section{Acknowledgements}

Sincere thanks are due to Dr. Abdul Kareem S. Al-Yassari of the College of Veterinary Medicine, University of AlQasim Green, Iraq, for to help with some bacterial analyses.

Conflict of interest: The authors declare that they have no conflict of interest.

Ethical approval: all applicable national and international guidelines for the care and use of animals were followed.

\section{References}

Adanir, D.O.R. \& Turutoglu, H. (2007). Isolation and antibiotic susceptibility of
Aeromonas hydrophila in a carp (Cyprinus carpio) hatchery farm. Bull. Vet. Inst. Pulawy., 51: 361-364.

guilera, A.M.G.; Hernande, C.C.; Zuniga, G.; Figueras, M.J. \& Castro, E.G. (2005). Aeromonas hydrophila clinical and environmental ecotypes as revealed by genetic diversity and virulence genes. FEMS Microbiol. Lett., 242(2): 231-240.

Albert, V. \& Ransangan, J. (2013). Effect of water temperature on susceptibility of culture marine fish species to vibriosis. Inter. J. Res. Pure Appl. Microbiol., 3: 48-52.

Al-Niaeem, K.S.; Shnawa, I.M.S. \& AlSa'adi, B.A. (2015). The immunological response for spleen as biomarker against of Aeromonas hydrophila bacterial in Cyprinus carpio fish. Basrah J. Vet. Res., 15(2): 306-315.

Al-Taee, A.M..R; Khamees, N.R. \& AlShammari, N.A.H. (2017). Vibrio species isolated from farmed fish in Basra City in Iraq. J. Aquac. Res. Dev., 8: 472. DOI: 10.4172/21559546.1000472.

Aoki T. (1999). Motile aeromonads (Aeromonas hydrophila). Pp: 427-453. In: Fish diseases and disorders. Woo PTK, Bruno D.W. (ed.) CABI Publishing, Wallingford: 944pp.

Aravenaromán, M.; Inglis, T.J.; Riley, T.V. \& Chang, B.J. (2014). Distribution of 13 virulence genes among clinical and environmental Aeromonas spp in Western Australia. European J. Clin. Microbiol. Inf. Dis., 33(11): 1889-1895. 
Austin, B. \& Austin, D.A. (2012). Aeromonadaceae representatives (motile aeromonads): 119-146. In: Austin, B. \& Austin, D.A. (Eds.), Bacterial fish pathogens, diseases of farmed and wild fish. $5^{\text {th }}$ ed. Springer, New York, London: 652pp.

Buján, N.; Romalde, J.L. \& Figueras, M.J. (2010). Comparison of phenotypical and genetic identification of Aeromonas strains isolated from diseased fish. Syst. Appl. Microbiol., 33: 149.153.

Beazhidalgo, R. \& Figueras, M.J. (2013). Aeromonas spp whole genomes and virulence factors implicated in fish disease. J. Fish Dis., 36(4): 371-388.

Bondad-Reantaso, M.G.; Subasinghe, R.P.; Arthur, J.R.; Ogawa, K.; Chinabut, S.; Adlard, R.; Tan, Z. \& Shariff, M. (2005). Disease and health management in Asian aquaculture. Vet. Parasitol., 132(3-4): 249-272.

Chopra, I. \& Roberts, M. (2001) Tetracycline antibiotics: mode of action, applications, molecular biology, and epidemiology of bacterial resistance. Microbiol. Mol. Biol., Rev., 65: 232260.

Cipriano, R.C. (2001). Aeromonas hydrophila and motile Aeromonad septicemias of fish. Fish Dis Leaflet 68, United States Department of the Interior fish and wildlife service, Div. Fish. Res. Washington, D.C. 25pp.

Dixon, B.A. \& Issvoran G. (1993). Antibacterial drug resistance in Aeromonas spp. isolated from domestic goldfish and koi from California. J. World Aquac. Soc., 24: 102-104.
El-Bouhy, Z.M.; El-Nobi, G.; Reda, R.M. \& Ali, S. (2015). Prevalence of septicemia and red mouth disease caused by Aeromonas sobria at Sahl ElHousinia fish farm. Zagazig Vet. J., 43(3): 53-63

Gao, Z.L.; Ji, X.; Guo, X.J.; Chen, P.; Liu, Y.J.; Liu, J. \& Sun, Y. (2016). Establishment and application of a duplex real time fluorescence quantitative PCR assay for detection of Aeromonas hydrophila. Chinese J. Zoonoses, 32(12): 1126-1130.

Guz, L. \& Kozinska, A. (2004). Antibiotic susceptibility of Aeromonas hydrophila and $A$. sobria isolated from farmed carp (Cyprinus carpio L.). Bull. Vet. Inst. Pulawy, 48: 391-395.

Hassan, A.; Noureldin, E.A.; Mahmoud, M. \& Fita, N.A. (2017). Molecular identification and epizootiology of Aeromonas veronii infection among farmed Oreochromis niloticus in Eastern Province, KSA. Egyptian J. Aqu. Res., 43: 161-167.

Janda, J.M. \& Abbott, S.L. (2010). The genus Aeromonas: taxonomy, pathogenicity and infection. Clin. Microbiol. Rev., 23: 35-73.

Ko, W.C.; Wu, H.M.; Chang, T.C.; Yan, J.J. \& Wu, J.J. (1998). Inducible ßlactam resistance in Aeromonas hydrophila: therapeutic challenge for antimicrobial therapy. J. Clin. Microbiol., 36: 3188-3189.

Li, F.; Wu, D. \& Gu, H.R. (2019). Aeromonas hydrophila and Aeromonas veronii cause motile Aeromonas septicaemia in the cultured Chinese sucker, Myxocyprinus asiaticus. Aqu. 
Res., 1-12. https:// doi.org/ 10.1111/ are. 14028

Nawaz, M.; Sung, K.; Khan, S.A., Khan, A.A. \& Steele, R. (2006). Biochemical and molecular characterization of tetracycline-resistant Aeromonas veronii isolates from catfish. Appl. Environ. Microbiol., 72(10): 6461-6466.

Pachanawan, A.; Phumkhachorn, P. \& Rattanachaikunsopon, P. (2008). Potential of Psidium guajava supplemented fish diets in controlling Aeromonas hydrophila infection in tilapia (Oreochromis niloticus). J. Biosci. Bioeng., 106(5): 419-424.

Rijnsdorp, A.D.; Peck, M.A.; Engelhard, G.H., Mollmann, C. \& Pinnegar, J.K. (2009). Resolving the effect of climate change on fish populations. ICES J. Mar. Sci., 66: 1570-1583.

Skwor, T.; Shinko, J.; Augustyniak, A.; Gee, C. \& Andraso, G. (2014). Aeromonas hydrophila and Aeromonas veronii predominate among potentially pathogenic ciprofloxacin and tetracycline resistant Aeromonas isolates from Lake Erie. Appl. Environmen. Microbiol., 80(3): 841848.

Soltan, D.M.M.; Yazdi, M.K.S \& Avadisians, S. (2012). Study of prevalence and antibiotic resistance in Aeromonas spp. isolated from minced meat and chicken samples in Iran. African J. Microbiol. Res., 6(7): 1568157. 